\title{
Calibrating Transcriptional Activity Using Constitutive Synthetic Promoters in Mutants for Global Regulators in Escherichia coli
}

\author{
Ananda Sanches-Medeiros, Lummy Maria Oliveira Monteiro, and Rafael Silva-Rocha \\ Systems and Synthetic Biology Lab, FMRP - University of São Paulo, Ribeirão Preto, SP, Brazil \\ Correspondence should be addressed to Rafael Silva-Rocha; silvarochar@usp.br
}

Received 24 October 2017; Accepted 30 January 2018; Published 21 March 2018

Academic Editor: João Paulo Gomes

Copyright (c) 2018 Ananda Sanches-Medeiros et al. This is an open access article distributed under the Creative Commons Attribution License, which permits unrestricted use, distribution, and reproduction in any medium, provided the original work is properly cited.

\begin{abstract}
The engineering of synthetic circuits in cells relies on the use of well-characterized biological parts that would perform predicted functions under the situation considered, and many efforts have been taken to set biological standards that could define the basic features of these parts. However, since most synthetic biology projects usually require a particular cellular chassis and set of growth conditions, defining standards in the field is not a simple task as gene expression measurements could be affected severely by genetic background and culture conditions. In this study, we addressed promoter parameterization in bacteria in different genetic backgrounds and growth conditions. We found that a small set of constitutive promoters of different strengths controlling a short-lived GFP reporter placed in a low-copy number plasmid produces remarkably reproducible results that allow for the calibration of promoter activity over different genetic backgrounds and physiological conditions, thus providing a simple way to set standards of promoter activity in bacteria. Based on these results, we proposed the utilization of synthetic constitutive promoters as tools for calibration for the standardization of biological parts, in a way similar to the use of DNA and protein ladders in molecular biology as references for comparison with samples of interest.
\end{abstract}

\section{Introduction}

Understanding the logic underlying the genetics of a microorganism based on the dynamics of its promoters and transcription factors is essential for manipulation of other living systems. A way to study this logic is introducing synthetic circuits provided with a reporter gene into living cells and analyzing the results of the expression $[1,2]$. However, the success of the implementation of complex circuits in living cells relies strongly on the correct production of the molecular components of the cells and is not limited to the influences of the promoters and transcription factors on gene expression. Several factors are responsible for controlling gene expression, including the rates of mRNA and protein production and their rates of degradation. However, synthesis of mRNA depends strongly on promoter strength, which determines how frequently the RNA polymerase (RNAP) is recruited to the promoter to initiate transcription [3]. On the other hand, the rate of protein production depends strongly on the strength of the ribosome binding site (RBS) in recruiting ribosomes for the translation of the target protein [4]. Additionally, the dilution or degradation of mRNA and proteins depends on the physiological state of the cell just as how their synthesis also relies on cell physiology with respect to the availability of nucleotides, amino acids, RNAP, and ribosomes [5]. In this way, changes in cell physiology and growth conditions can cause variability in gene expression in a manner that is independent of promoter regulation [5].

On account of these possible variations between the cells, several attempts have been made to establish biological standards for promoter activity, and the use of internal promoters as references has been proposed some years ago $[6,7]$. More recently, the use of calibrated internal promoters has been proposed as an alternative for defining relative promoter activities during experimental measurements of transcription levels. In this method, an endogenous (or reference) promoter is placed in the same plasmid as the target promoter, each of them controlling the expression of a different fluorescent protein, and the intrinsic promoter activity is 
calculated as a ratio of the two outputs [8]. However, the expression of additional genes in the host bacterium can increase genetic load and influence gene expression as well. In this way, inserting a calibrated internal promoter would disturb cell functions [9]. Additionally, most methods have focused on the analysis of maximal promoter activity at fixed conditions or on linear expression range of promoter activity, limiting the utilization of standards on condition where cells are subjected to changing physiological regimens [8]. These requirements make the use of calibration methods for the analysis of regulated promoters extremely difficult.

In this study, we seek to analyze intrinsic promoter activity using a single reporter gene in different strains of Escherichia coli by using a simple and straightforward protocol. For the determination of intrinsic promoter activity, we used a low-copy number plasmid based on the p15a origin of replication (ori) and a short-lived GFP with LVA tag [10]. We analyzed four constitutive promoters available in the Registry of Standard Biological Parts and a wild-type Plac promoter as regulated system. As hosts, we used two strains of E. coli with mutant global regulatory proteins, ihf and fis, which are responsible for regulating the expression of hundreds of genes in this bacterium [11], obtained from the widely used Keio collection of E. coli mutants [12]. Additionally, glucose was used as the external source of variation, since all strains exhibited improved growth rates in its presence. Under the conditions of the analysis, we observed that the system we had used exhibited invariant promoter activities that were independent of the strains and growth conditions used, indicating it was able to demonstrate the intrinsic properties of the promoters analyzed. In addition, to prove that our calibrator works, we tested the natural promoter of Pseudomonas putida Pm promoter with different concentrations of 3-methylbenzoate $(3 \mathrm{MBz})$ [13] and calibrate it with our four constituent promoters in liquid and solid medium. In this way, it was possible to verify that the calibrator works and presents a potential application in synthetic biology. In this regard, we propose a simple, plasmid-based and single reporter method for promoter calibration that is compatible for use with regulated promoters and changes in growth conditions, which could be fundamental to the characterization of biological parts in synthetic biology.

\section{Material and Methods}

2.1. Bacterial Strains, Plasmids, and Growth Conditions. The bacterial strains, plasmids, and primers used in this study are listed in Table 1. E. coli $\mathrm{DH} 5 \alpha$ was used for cloning the pMR1-Pjx (where $x$ stands for 100,106, 114, and 113) and pMR1-Plac vectors [14] by transformation using the heatshock method, and E. coli DH10B was used for cloning pGLR2-Pjx vectors by electroporation and for GFP/Lux expression analysis [15]. For the calibration of promoter activity, E. coli BW25113 was used as wild-type strain, and ihf ( $\Delta i h f)$ or fis ( $\Delta$ fis) mutants (from the Keio collection) were used as mutant hosts with reduced growth rate. Plasmids pMR1 and pGLR2 were used as reporter systems for promoter analysis. Plasmid pMR1 has a low-copy p15a ori, a chloramphenicol-resistance marker, and two genes encoding fluorescent proteins oriented in opposite directions ( $m$ Cherry and gfplva). Plasmid pGLR2 has a low-copy RK2 origin of replication, a kanamycin resistance marker, and two reporter genes oriented in the same direction, namely, the GFP gene followed by luxCDABE. Although the vector has GFP, in this work when pGLR2 was used, only the Lux was measured. The E. coli strains were grown at $37^{\circ} \mathrm{C}$ with aeration at an agitation rate of $180 \mathrm{rpm}$ in LB medium (for overnight growth) or $\mathrm{M} 9$ minimal medium (containing $6.4 \mathrm{~g} / \mathrm{L} \quad \mathrm{Na}_{2} \mathrm{HPO}_{4} \bullet 7 \mathrm{H}_{2} \mathrm{O}, 1.5 \mathrm{~g} / \mathrm{L}_{2} \mathrm{KH}_{2} \mathrm{PO}_{4}, 0.25 \mathrm{~g} / \mathrm{L} \mathrm{NaCl}$, and $0.5 \mathrm{~g} / \mathrm{L} \mathrm{N} \mathrm{NH}_{4} \mathrm{Cl}$ ) supplemented with $2 \mathrm{mM} \mathrm{MgSO}_{4}$, $0.1 \mathrm{mM} \mathrm{CaCl}, 0.1 \mathrm{mM}$ casamino acids, and $1 \%$ glycerol as the sole carbon source (for growth during the analysis). When required, chloramphenicol $(34 \mu \mathrm{g} / \mathrm{mL})$, kanamycin $(50 \mu \mathrm{g} / \mathrm{mL})$, or glucose $(0.4 \%)$ was added to the medium. In the minimal medium, the antibiotics were added at half of the previously mentioned concentrations.

2.2. Plasmid and Strain Construction. For the experiments, oligonucleotides were synthesized (Exxtend, Campinas, Brazil) based on the synthetic constitutive promoters from the iGEM BBa_J23104 set of promoters (http://parts.igem. org/Part:BBa_J23104), with an annealing site on PMR1 and restriction sites for EcoRI and BamHI. The promoters J23100, J23106, J23114, and J23113 (referred here as Pj100, Pj106, Pj114, and Pj113, resp.) were used (see Table 1). Once these fragments were amplified by PCR, they were digested by EcoRI and BamHI and inserted into the multiple cloning sites (MCS) of pMR1 and pGLR2, and thus, generating pMR1-Pjx and pGR2-Pjx. These plasmids were inserted into DH5 $\alpha$ and DH10B strains, respectively, cloned, and sequenced. The plasmids, pMR1-Pjx were inserted into $E$. coli BW25113 and into ihf and fis mutants obtained from the Keio collection.

The xylS, PxylS, and Pm promoters were PCR amplified with Phusion High-Fidelity DNA polymerase (Thermo Fisher Scientific) using the primer pairs 5_xylS_EcoRI $\left(5^{\prime}\right.$-GAA TTC TCA AGC CAC TTC CTT TTT GCA TTG-3 $\left.{ }^{\prime}\right)$ and 3_Pm_BamHI ( $5^{\prime}$-GGA TCC ATT ATT GTT TCT GTT GCA TAA AGC C-3') and pSEVA438 vector (pBBR1 replication origin, Sm/Sp; Silva-Rocha and de Lorenzo [1]) as template. These primers introduced EcoRI and BamHI restriction sites (underlined) at the $5^{\prime}$ and $3^{\prime}$ ends, respectively. The PCR products were gel purified, digested with EcoRI/BamHI, and ligated to the pMR1 vector previously cut with the same restriction enzymes. The resulting plasmids were sequenced to check integrity and inserted to E. coli strains (E. coli BW25113and into ihf and fis mutants). The resulting plasmid was named pMR1-xylS-Pm.

2.3. GFP Fluorescence and Bioluminescence Assays and Data Processing. To analyze promoter activity, single colonies of recombinant strains containing pMR1-Pjx and E. coli DH10B containing pGLR2-Pjx were grown overnight in LB medium that was supplemented with chloramphenicol $(34 \mu \mathrm{g} / \mathrm{mL})$ or kanamycin $(50 \mu \mathrm{g} / \mathrm{mL})$ for plasmid selection at $37^{\circ} \mathrm{C}$ with aeration and agitated at $180 \mathrm{rpm}$. The strains 
TABLE 1: Strains, plasmids and primers used in this study.

\begin{tabular}{|c|c|c|}
\hline Strains, plasmids, and primers & Description & Reference \\
\hline \multicolumn{3}{|l|}{ Strains } \\
\hline E. coli $\mathrm{DH} 5 \alpha$ & $\begin{array}{c}\mathrm{F}^{-} \text {endA1 glnV44 thi-1 recA1 relA1 gyrA96 deoR nupG purB20 } \varphi 80 \mathrm{~d} l a c Z \Delta \mathrm{M} 15 \\
\Delta(\operatorname{lacZYA}-\operatorname{argF}) \mathrm{U} 169, \mathrm{hsdR} 17\left({r_{K}^{-}}^{-} m_{K}^{+}\right), \lambda^{-}\end{array}$ & {$[12]$} \\
\hline E. coli $\mathrm{DH} 10 \mathrm{~B}$ & $\begin{array}{c}m c r A \Delta m r r-h s d R M S-m c r B C) \varphi \text { 80lacZ } \Delta M 15 \Delta l a c X 74 \text { recA1 araD139 } \Delta \\
\text { (ara-leu)7697 galU galK rpsL endA1 nupG } \Delta d c m .\end{array}$ & {$[12]$} \\
\hline E. coli BW25113 & $\begin{array}{l}\text { lacI }^{+} r r n B_{\mathrm{T} 14} \Delta l a c Z_{\mathrm{WJ16}} h s d R 514 \Delta a r a B A D_{\mathrm{AH} 33} \Delta r h a B A D_{\mathrm{LD} 78} r p h-1 \\
\Delta(a r a B-D) 567 \Delta(r h a D-B) 568 \Delta l a c Z 4787(:: r r n B-3) h s d R 514 r p h-1 .\end{array}$ & {$[12]$} \\
\hline E. coli JW1702 & E. coli BW25113 $\Delta$ ihf mutant & {$[12]$} \\
\hline E. coli JW3229 & E. coli $B W 25113 \Delta$ fis mutant & {$[12]$} \\
\hline \multicolumn{3}{|l|}{ Plasmids } \\
\hline pMR1 & $\mathrm{Cm}^{\mathrm{R}}$, ori p15a. GFPlva promoter probe vector & {$[14]$} \\
\hline pMR1-Pj113 & pMR1 with $P j 113$ cloned as EcoRI/BamHI fragment & This work \\
\hline pMR1-Pj114 & pMR1 with Pj114 cloned as EcoRI/BamHI fragment & This work \\
\hline pMR1-Pj106 & pMR1 with Pj106 cloned as EcoRI/BamHI fragment & This work \\
\hline pMR1-Pj100 & pMR1 with $P j 100$ cloned as EcoRI/BamHI fragment & This work \\
\hline pMR1-Plac & pMR1 with Plac promoter cloned as EcoRI/BamHI fragment & [28] \\
\hline pGLR2 & $\mathrm{Km}^{\mathrm{R}}$, oriT, ori $\mathrm{RK} 2$. SEVA-based vector with dual GFP-lux reporter & [15] \\
\hline pGLR2-Pj113 & pGLR2 with $P j 113$ cloned as EcoRI/BamHI fragment & This work \\
\hline pGLR2-Pj114 & pGLR2 with Pj114 cloned as EcoRI/BamHI fragment & This work \\
\hline pGLR2-Pj106 & pGLR2 with $P j 106$ cloned as EcoRI/BamHI fragment & This work \\
\hline pGLR2-Pj100 & pGLR2 with Pj100 cloned as EcoRI/BamHI fragment & This work \\
\hline pMR1-xylS-Pm & pMR1 with PxylS, xylS and $P m$ cloned as EcoRI/BamHI fragment & This work \\
\hline \multicolumn{3}{|l|}{ Primers } \\
\hline Pj100-FW & GAATTCTTGACGGCTAGCTCAGTCCTAGG & This work \\
\hline Pj100-RV & TACAGTGCTAGCAAGTGGATCCTTGCGATC & This work \\
\hline Pj106-FW & GAATTCTTTACGGCTAGCTCAGTCCTAGGTA & This work \\
\hline Pj106-RV & TAGTGCTAGCAAGTGGATCCTTGCGATC & This work \\
\hline Pj114-FW & GAATTCTTTATGGCTAGCTCAGTCCTAGGT & This work \\
\hline Pj114-RV & ACAATGCTAGCAAGTGGATCCTTGCGATC & This work \\
\hline Pj113-FW & GAATTCCTGATGGCTAGCTCAGTCCTAGGG & This work \\
\hline Pj113-RV & ATTATGCTAGCAAGTGGATCCTTGCGATC & This work \\
\hline 5_xylS_EcoRI & GAATTCTCAAGCCACTTCCTTTTTGCATTG & This work \\
\hline 3_Pm_BamHI & GGATCCATTATTGTTTCTGTTGCATAAAGCC & This work \\
\hline
\end{tabular}

grown overnight were washed with $\mathrm{MgSO}_{4}(10 \mathrm{mM})$ buffer, resuspended in the same buffer, and diluted to a ratio of 1:20 with $\mathrm{M} 9$ minimal medium (containing $6.4 \mathrm{~g} / \mathrm{L} \mathrm{Na}{ }_{2} \mathrm{H}$ $\mathrm{PO}_{4} \bullet 7 \mathrm{H}_{2} \mathrm{O}, 1.5 \mathrm{~g} / \mathrm{L} \mathrm{KH}_{2} \mathrm{PO}_{4}, 0.25 \mathrm{~g} / \mathrm{L} \mathrm{NaCl}$, and $0.5 \mathrm{~g} / \mathrm{L}$ $\mathrm{NH}_{4} \mathrm{Cl}$ ) supplemented with $2 \mathrm{mM} \mathrm{MgSO}_{4}, 0.1 \mathrm{mM} \mathrm{CaCl}_{2}$, $0.1 \mathrm{mM}$ casamino acids, chloramphenicol $(17 \mu \mathrm{g} / \mathrm{mL})$, and $1 \%$ glycerol as the sole carbon source. When required, glucose $(0.4 \%)$ was supplemented to the medium. In total, $200 \mu \mathrm{L}$ of the culture was placed in a 96-well plate and analyzed using a Victor X3 plate reader (PerkinElmer) over several hours at $37^{\circ} \mathrm{C}$. At 30 -minute time intervals, the optical density at $600 \mathrm{~nm}\left(\mathrm{OD}_{600 \mathrm{~nm}}\right)$ and the fluorescence (excitation $485 \mathrm{~nm}$ and emission $535 \mathrm{~nm}$ ) were measured for the strains containing pMR1-Pjx; the optical density at $600 \mathrm{~nm}\left(\mathrm{OD}_{600 \mathrm{~nm}}\right)$, the fluorescence (excitation $485 \mathrm{~nm}$ and emission $535 \mathrm{~nm}$ ), and the bioluminescence were measured for DH10B containing pGLR2-Pjx. Promoter activities were expressed as fluorescence or bioluminescence normalized by the $\mathrm{OD}_{600 \mathrm{~nm}}$ upon background normalization (fluorescence/OD $600 \mathrm{~nm}$ ). As a positive control for pMR1-Pjx analysis, wild type lac promoter (Plac), which is regulated by CRP, was used. Data analysis and representation was performed using Microsoft Excel (2016) and ad hoc $R$ script. To prove that the pMR1-Pjx system works as a gene expression standard, the natural promoter of $P$. putida $P m$, which is regulated by $x y l S$ when this regulator is induced by $3 \mathrm{MBz}$, was used [13]. The pMR1-xylS-Pm construct contains the $x y l S$ promoter $(P x y l S)$, which in the presence of $3 \mathrm{MBz}$ leads to the expression of the XylS regulatory protein. The XylS regulator binds to $3 \mathrm{MBz}$ and activates the $\mathrm{Pm}$ promoter by inducing the expression of GFP. Data analysis and representation were performed using ad hoc $R$ script. 


\section{Results and Discussion}

3.1. Quantification of Constitutive Promoter Activity Using GFP and luxCDABE Reporters. For the analysis of promoter activities, we used two reporter plasmids based on a shortlived GFP variant placed into a narrow-host-range vector (pMR1 [14]) and a synthetic GFP-luxCDABE reporter system placed into a broad-host-range vector $[15,16]$ to measured Lux, as represented in Figure 1(a). In order to observe the effects of these differences (regarding use of different reporter systems) on the measurement of the activities of the promoters of interest, we analyzed the promoter activities of four BioBrick parts, namely Pj100, Pj106, $\mathrm{Pj} 114$, and $\mathrm{Pj} 113$, that contain mutations in the sequences at -35 or -10 and exhibit about $100 \%, 50 \%, 10 \%$, and $1 \%$ activities, respectively (relative to $\mathrm{Pj} 100$ activity). As shown in Figures 1(b) and 1(c), maximal promoter activity of the four synthetic promoters analyzed were virtually identical for both the short-lived GFPlva and the luxCDABE reporters, resulting in relative activity values that are closer to the expected value. When we analyzed the promoter activities during the growth period of E. coli using the GFPlva and Lux reporters, we observed that the differences were present throughout the growth period of the bacteria, with better differentiation of the intrinsic promoter activities achieved using the luminescent reporter system (Figures 1(d) and $1(\mathrm{e})$ ). Although the luciferase reporter provided better differentiation, GFP reporter allows uses to perform single-cell experiments that cannot be made using light-emitting reporters. Since most synthetic biology works use the GFP reporter, and moreover, GFP provided sufficient resolution to analyze the promoters and also allowed for single-cell analysis (a possible calibrator approach), we focused on the section on the pMR1 reporter system containing the shortlived variant of the reporter protein.

\subsection{Robust Calibration of Promoter Activities under Different} Pleiotropic and Growth Conditions. During the experiments, wild type and mutant strains of E. coli were grown in minimal M9 medium with $1 \%$ glycerol and $1 \%$ glycerol plus $0.4 \%$ glucose in order that the cells were adapted to a richer physiological regimen. Figure 2(a) represents the critical steps in gene expression that were influenced by the bacterial hosts and growth conditions used. In this regard, the rate of mRNA synthesis $(\beta m)$ was the main parameter controlled by a specific synthetic promoter, while the rate of protein synthesis $(\beta p)$ was dependent on the strength of the RBS involved (which was the same in all constructs analyzed). Additionally, the rates of mRNA and protein degradation were dependent on the nature of the reporter sequence (i.e., due to differences in the sequence of reporter genes) and the growth rate of the bacteria (since fast-growing bacteria have higher dilution rates of mRNA and protein than slowgrowing bacteria). In this method, the use of constitutive promoters with different strengths allowed for variations in $\beta m$ and facilitated the analysis of its sensitivity to changes in the dilution or degradation rates of $\mathrm{mRNA}$ and proteins. As shown in Figures 2(b) and 2(c), both ihf and fis mutants exhibited reduced growth compared to the wild type for all constructs analyzed. However, in all cases, the addition of $0.4 \%$ glucose to the growth medium resulted in a stepwise improvement in the growth of the strains. In other words, in the presence of glucose, bacterial growth is faster, although there is no glucose effect on the final promoter activity. Our calibrator approach is an interesting way to avoid the genetic background differences, indicating that the calibrator can be used in several conditions to standardize promoter studies using different strains under a growth condition variety (glucose or $3 \mathrm{MBz}$-performed below).

In order to observe the effects of the differences in strains and growth medium on the measurements of the activities of the promoters of interest, we analyzed four synthetic promoters that contain sequence differences at -35 or -10 (Figure 3(a)). As shown in Figure 3(b), the regulated Plac promoter (a natural promoter used as reference) exhibited strong activity in the three strains analyzed (wild type, $\Delta i h f$, and $\Delta f i s$ ), and this activity was fully suppressed in the presence of glucose (due to the inactivation of CRP [17]). When we analyzed promoter activity of the four synthetic promoters, we observed that the promoter dynamics and steady state promoter activity were almost invariant in the different mutant strains and under the two growth conditions (Figure $3(\mathrm{c})$ ), indicating that these promoters were not influenced by the drastic physiological variations regarding the different strains of E. coli (E. coli BW25113, and into ihf and fis mutants). It is noteworthy that the same was observed for the addition of glucose to the medium that did not compromise the promoter activity. Again showing that the internal calibrator can be used in different situations. When we performed a comparison of the observed promoter activities, Pj106 exhibited an activity level very close to the expected value ( $45.7 \%$ observed versus $47 \%$ expected), whereas Pj114 and $P 113$ exhibited promoter activities varying by $3 \%$ and $2.3 \%$, respectively, from the value of the activity exhibited by Pj100 (compared to the expected values, $10 \%$ and $1 \%$, resp.). These expected values come from the previous analysis made by iGEM BBa_J23104 set of promoters (http://parts.igem.org/Part:BBa_J23104). These differences are due to the differences in the reporter, plasmids, and strains used for promoter characterization. Additionally, the Plac promoter exhibited activity of value about $30 \%$ under nonrepressive conditions when compared to Pj100 reference promoter.

These results show that the use of the short-lived GFP reporter in combination with constitutive promoters is a simple way to calibrate promoter activity under userspecific experimental conditions, similar to the way that DNA and protein ladders are used in molecular biology techniques as references for the comparison of specific targets. In conclusion, our data shows how intrinsic promoter activity can be calibrated using single reporter genes and simple data processing without the need for using internal promoter references.

3.3. The Calibrator Can Be Applied to the Induction System $x y l S-P m$. In order to prove that the set of four promoters proposed in this work acts as an internal calibrator even when applied to an induced expression system, we analyzed 


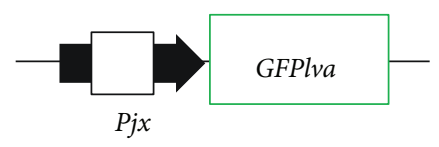

pMR1-Pjx

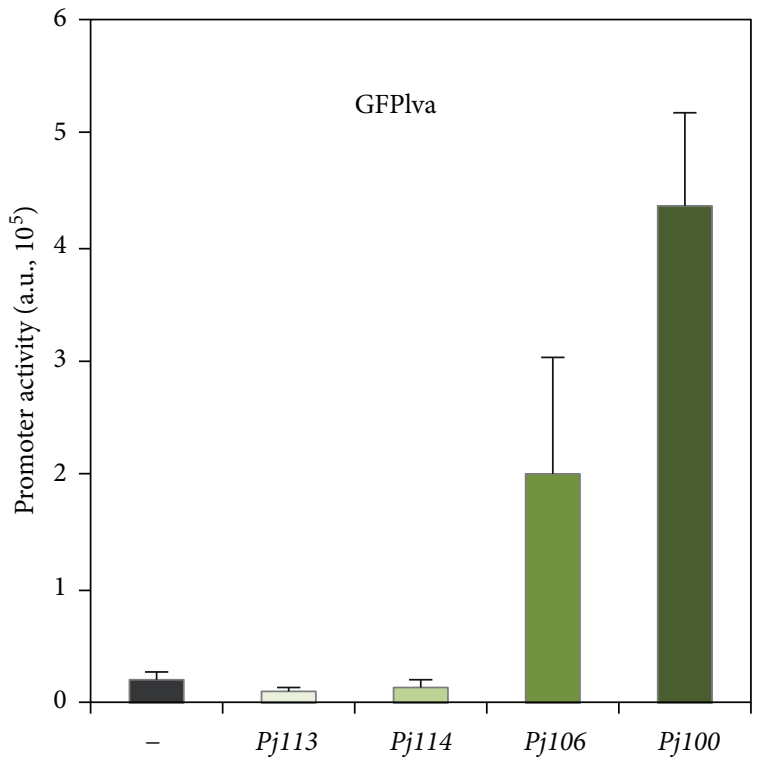

(b)

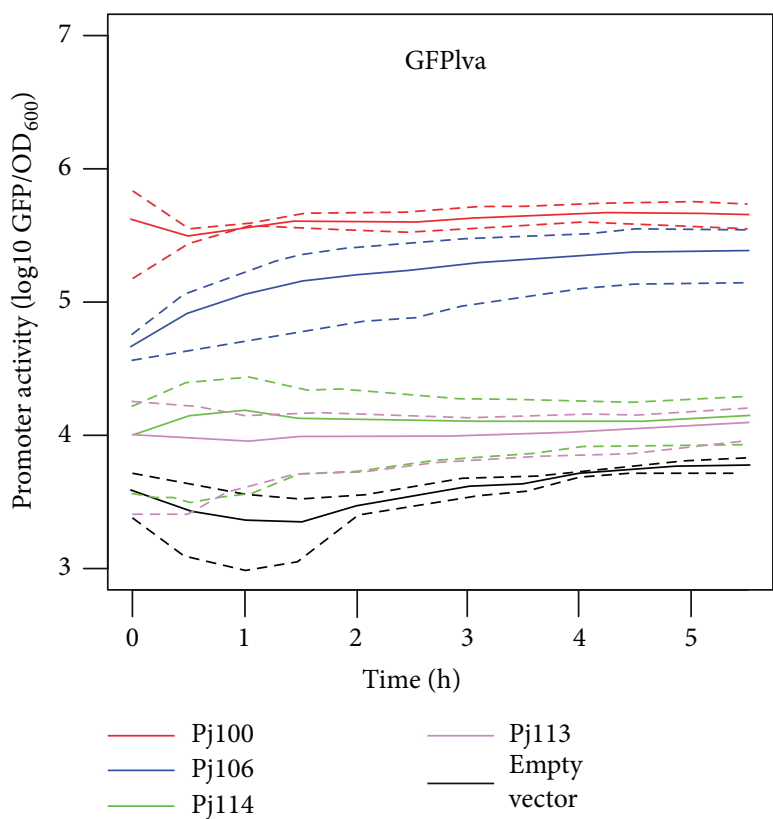

(d)

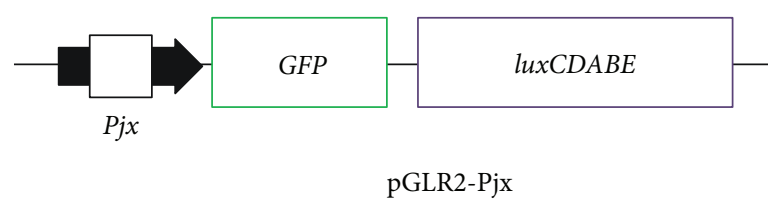

(a)

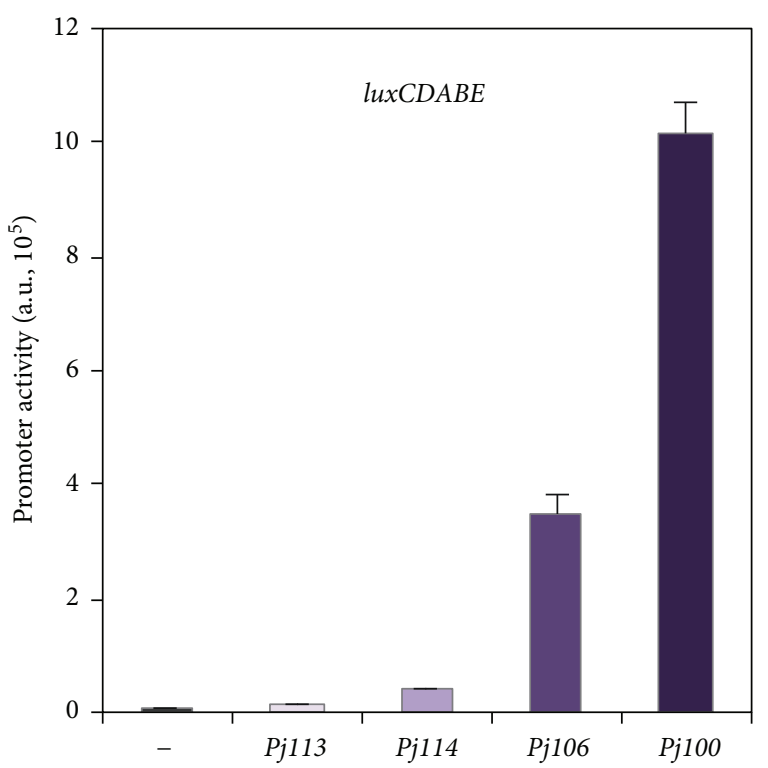

(c)

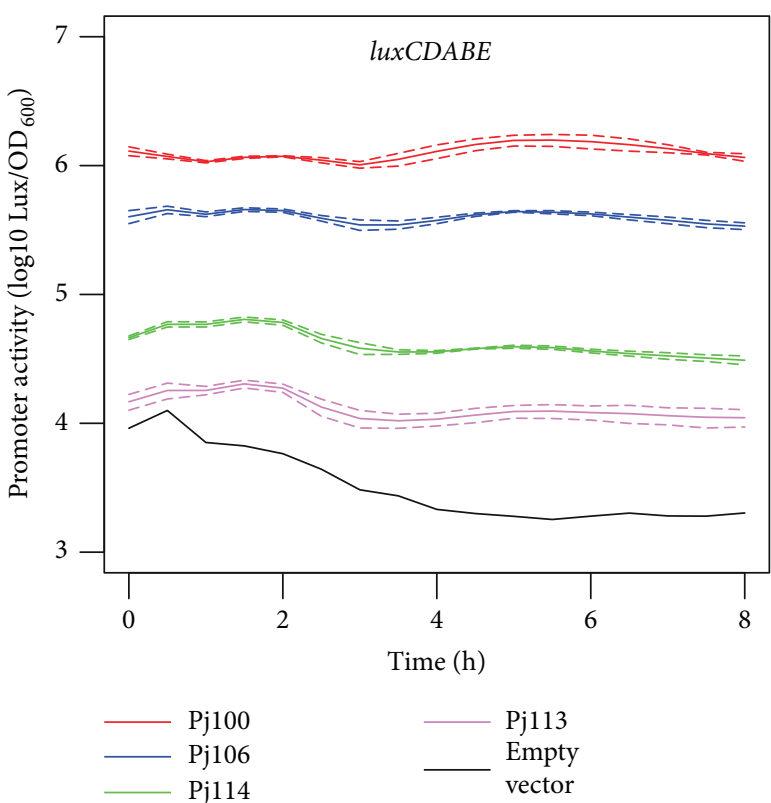

(e)

FIgURE 1: Construction and validation of the reporter systems. (a) Synthetic promoters were cloned into the plasmid pMR1, which contains a short-lived GFPlva variant, and pGLR2, a broad host range vector containing a GFP-luxCDABE reporter system. (b) Maximal promoter activity of the four promoters in pMR1 vector. (c) Maximal promoter activity analyzed by monitoring lux expression using pGLR2 constructions. (d) GFP expression profile along the growth curve from reporters cloned in pMR1 vector. (e) lux expression profile along the growth curve from reporters cloned in pGLR2 vector. The solid lines represent the average values calculated using data from three independent experiments while dashed lines represent standard deviation from the samples. 

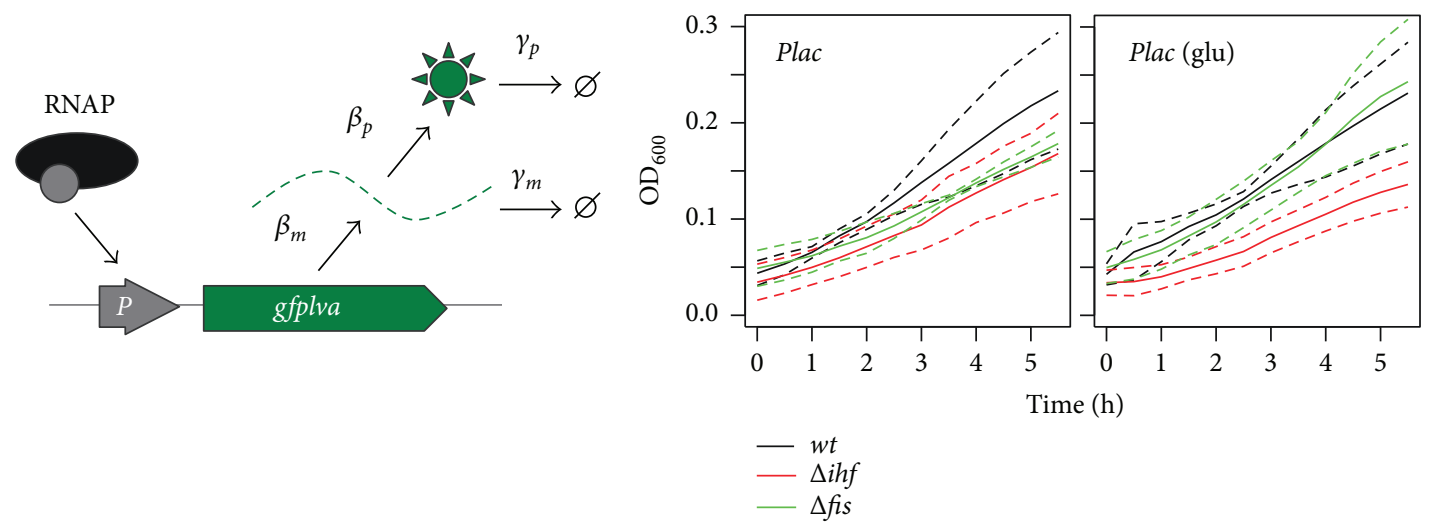

(a)

(b)
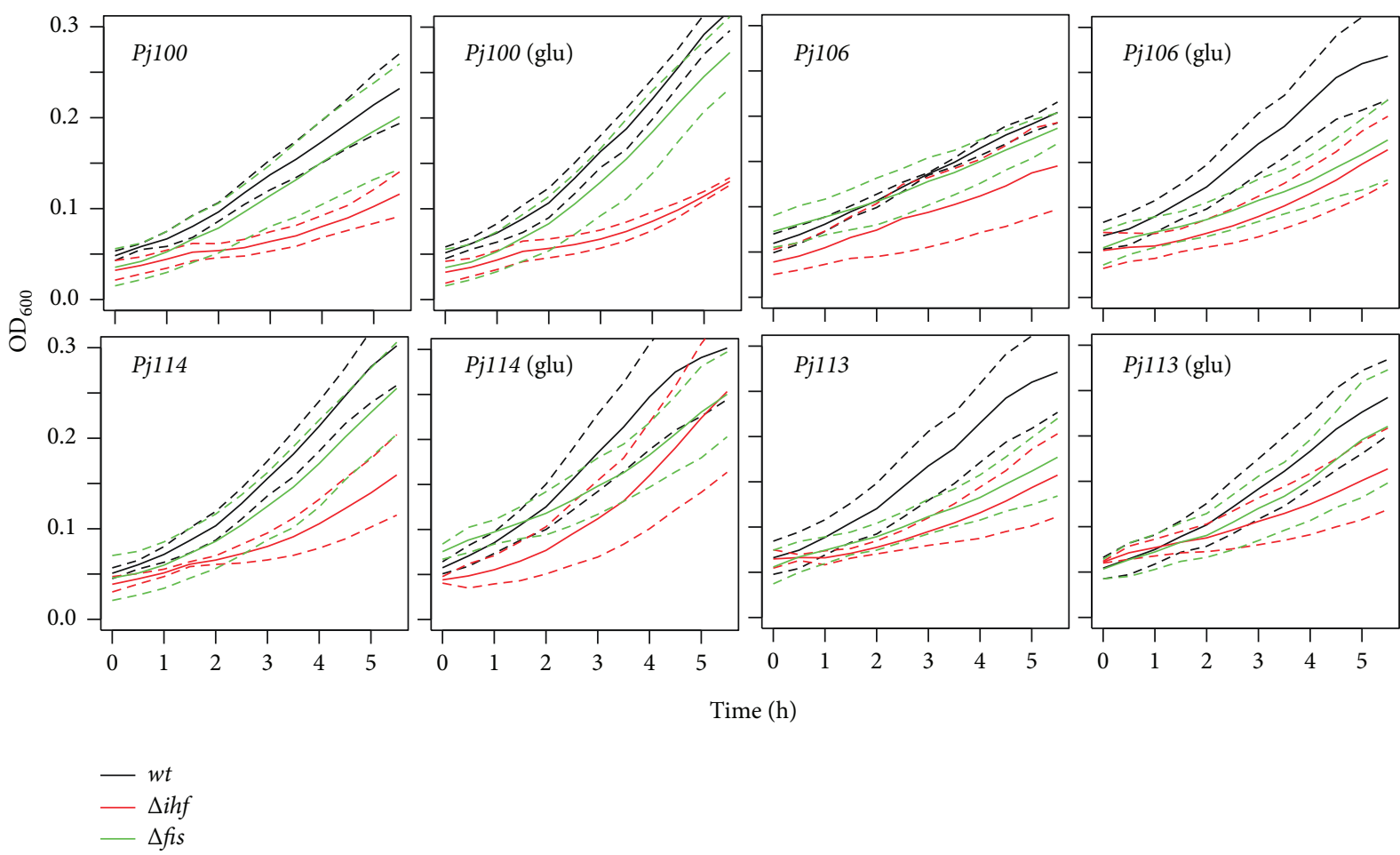

Time (h)

$$
\Delta i h f
$$

(c)

Figure 2: Quantification of growth variation in different E. coli strains under two physiological regimens. (a) Schematic representation of the main steps for gene expression in bacteria. The strength of the interaction between RNA polymerase (RNAP) and target promoter determines the rate of mRNA synthesis $\left(\beta_{m}\right)$, while the RBS sequence determines the rate of protein translation $\left(\beta_{p}\right)$. The rates of mRNA and protein dilution or degradation $\left(\gamma_{m}\right.$ and $\gamma_{p}$, resp.) depends on cell growth and physiological regimens of the cells. (b) Growth curve of E. coli strains harboring a Plac:: GFPlva fusion in minimal medium with $1 \%$ glycerol (left) or $1 \%$ glycerol plus $0.4 \%$ glucose (right) as carbon source. (c) Growth curve of E. coli strains harboring different promoter fusions (Pj100, Pj106, Pj114, and Pj113) in minimal medium with $1 \%$ glycerol or $1 \%$ glycerol plus $0.4 \%$ glucose (labeled as glu) as carbon source. Solid lines represent average values calculated using data from three independent experiments for wild type (black), $\Delta i h f$ (red), and $\Delta f i s$ (green) strains, while dashed lines represent the upper and lower limits of standard deviations.

the four synthetic promoters and pMR1-xylS-Pm system (Figure $4(\mathrm{a})$ ) with increasing concentrations of $3 \mathrm{MBz}$. In order to demonstrate that our calibrator is robust and even works in a blue light transilluminator, we analyzed colonies grown on petri dish contend medium $\mathrm{LB}$ plus $3 \mathrm{MBz}$ $1000 \mu \mathrm{M}$. As shown in Figure 4(b), pMR1-xylS-Pm displayed the same promoter activity as pMR1-P106. Next, we tested the system at increasing $3 \mathrm{MBz}$ inductor concentrations; they were carried out on the three E. coli strains previously used in this work. The data shown in Figure 4(d) are relative to 4.5 hours after the start of the induction. From Figure 4, it is possible to note that the increasing concentration of $3 \mathrm{MBz}$ did not promote differences in Pjx promoter activity (Figure 4(d)), neither during the 8 hours of the experiment (Figure 4(c)). On Figure 4(c), it is important to note that each color on the graph represents a different Pjx synthetic 

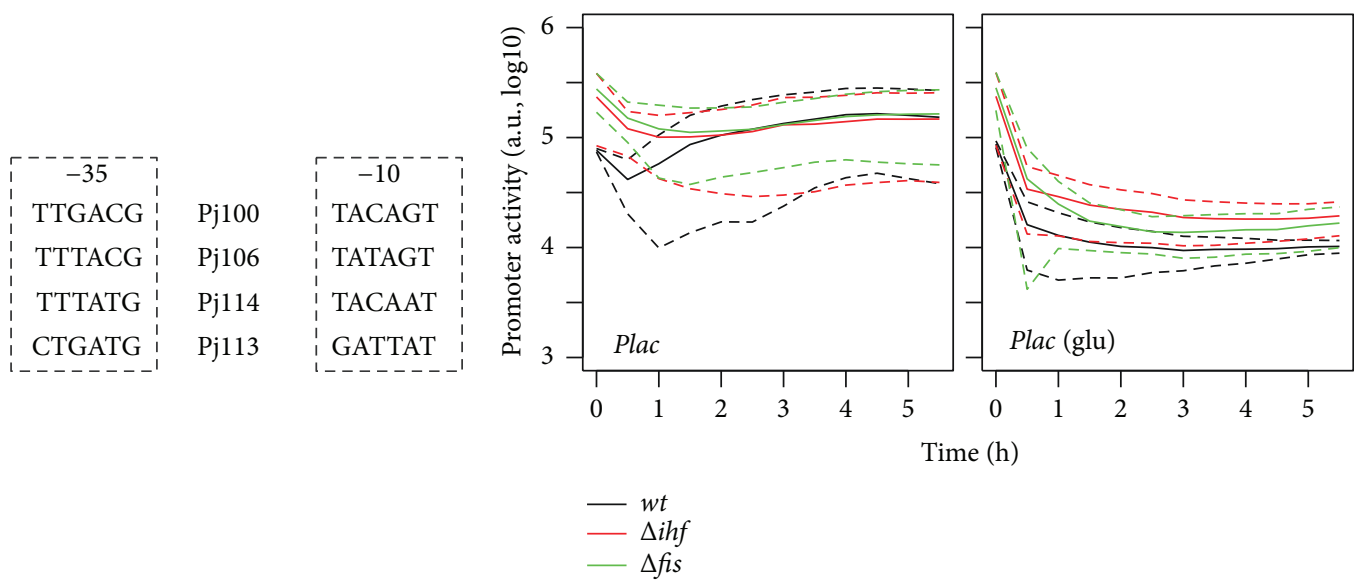

(a)

(b)
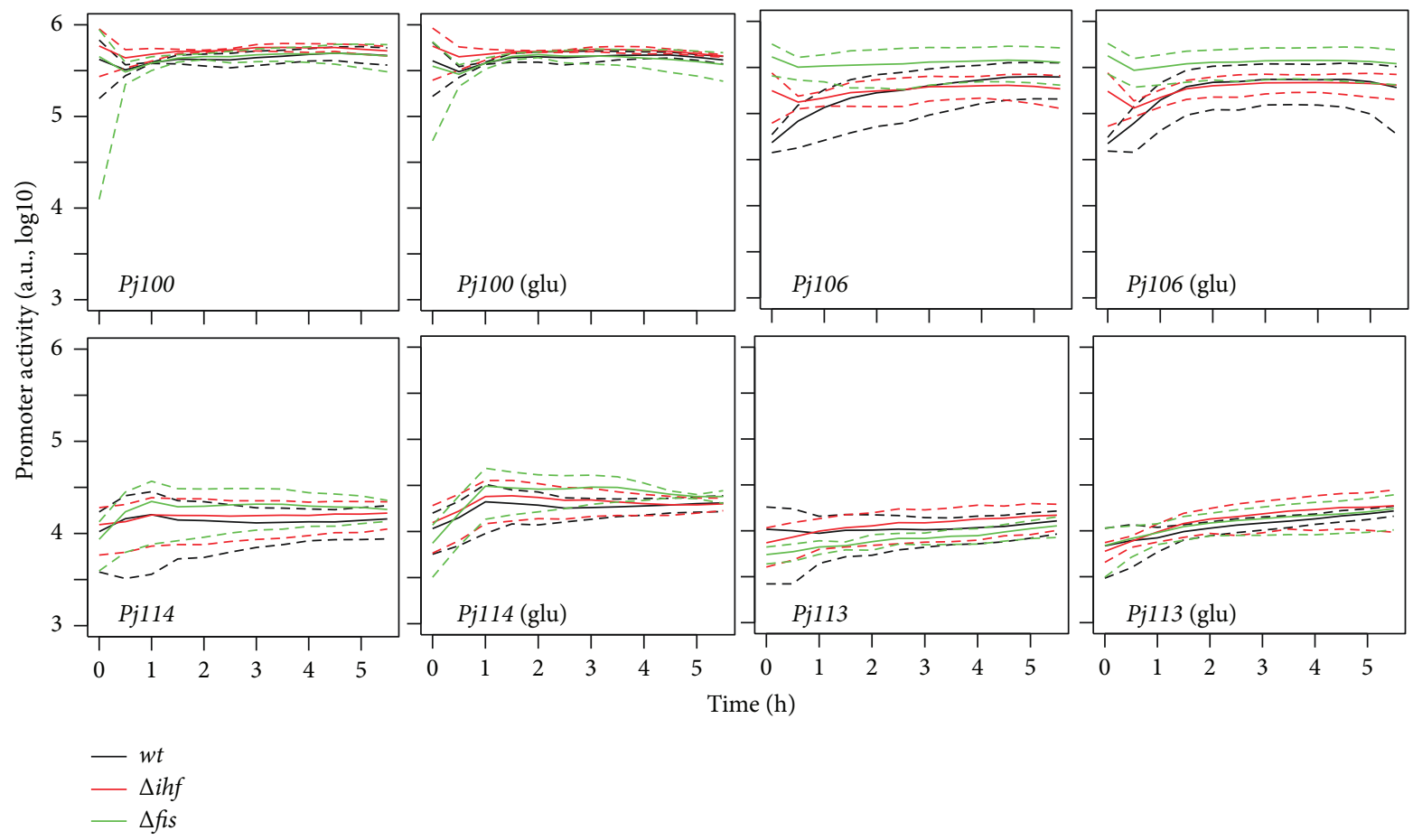

(c)

Figure 3: Quantification of promoter activities in different E. coli strains. (a) Representation of the sequences at -10 or -35 for the four constitutive promoters analyzed, using bold letters for bases conserved related to Pj100 reference. (b) Promoter activity of E. coli strains harboring a Plac::GFPlva fusion in minimal medium with $1 \%$ glycerol (left) or $1 \%$ glycerol plus $0.4 \%$ glucose (right) as carbon source. (c) Promoter activity of E. coli strains harboring different promoter fusions (Pj100, Pj106, Pj114, and Pj113) in minimal media with $1 \%$ glycerol or $1 \%$ glycerol plus $0.4 \%$ glucose (labeled as glu) as carbon source. Solid lines represent the average values calculated using data from three independent experiments for wild type (black), $\Delta i h f$ (red), and $\Delta$ fis (green) strains, while dashed lines represent the upper and lower limits of standard deviations.

promoter and the set of lines belonging to each color group refers to different $3 \mathrm{MBz}$ concentrations. In this sense, it is possible to note that there are no differences between the set color lines. This result suggests that the $3 \mathrm{MBz}$ addition do no promote differences on promoter activity. On the other hand, for a $3 \mathrm{MBz}$-induced system, the aromatic compound produced a change in promoter activity for a sigmoidal curve, proportional to the $3 \mathrm{MBz}$ increase concentration (Figure 4(d)).
A brief and simple conclusion can be made from Figure 4(d), regardless of the host strain used, in the range of 1 to $10 \mu \mathrm{M}$ ( 0 to 1 on the $\mathrm{x}$ axis) concentration, the Pm promoter presents similar promoter activity to $\mathrm{Pj} 114$ promoter. On the other hand, in the range of 10 to $100 \mu \mathrm{M}$ ( 1 to 2 on the $\mathrm{x}$-axis) concentration, $\mathrm{Pm}$ presents intermediate promoter activity to $P j 114$ and Pj106 promoters. Finally, in the range of 100 to $1000 \mu \mathrm{M}$ (2 to 3.0 on the $x$-axis) concentrations, $P m$ presents promoter activity close 


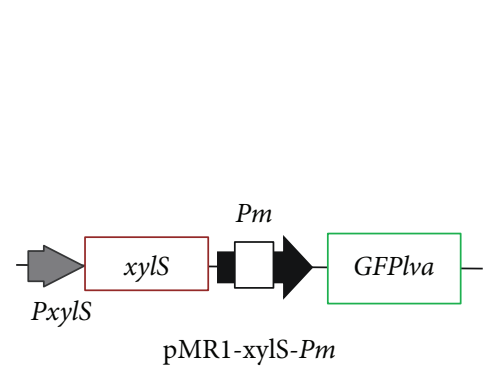

(a)

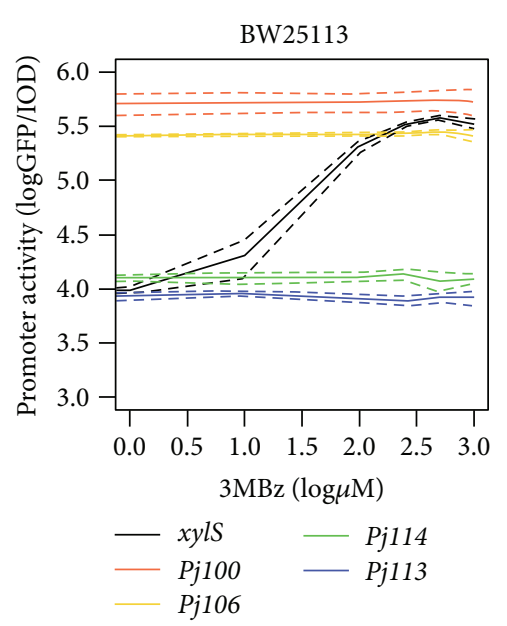

BW25113 3MBz $1000 \mu \mathrm{m}$
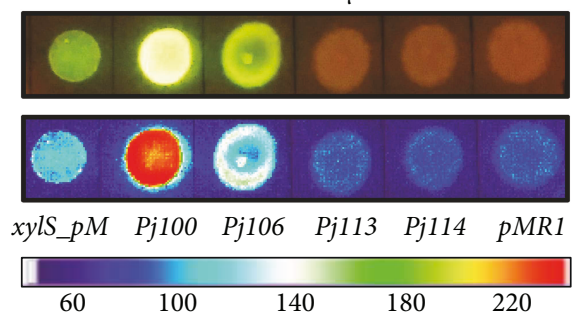

(b)

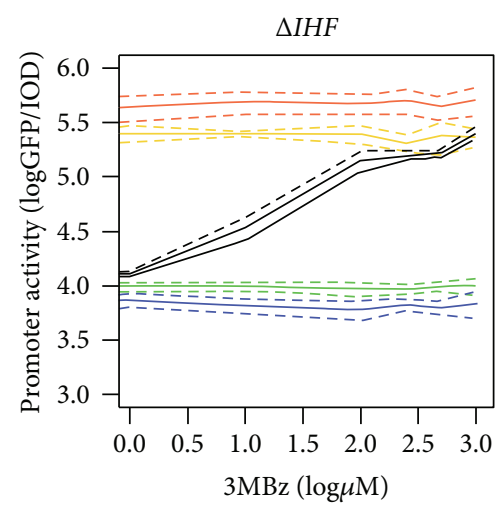

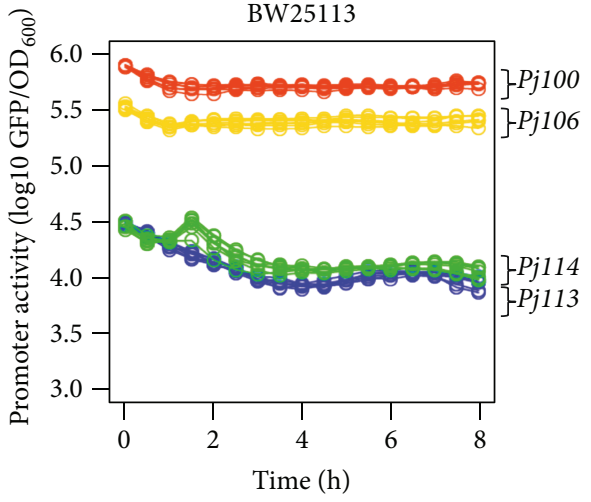

(c)

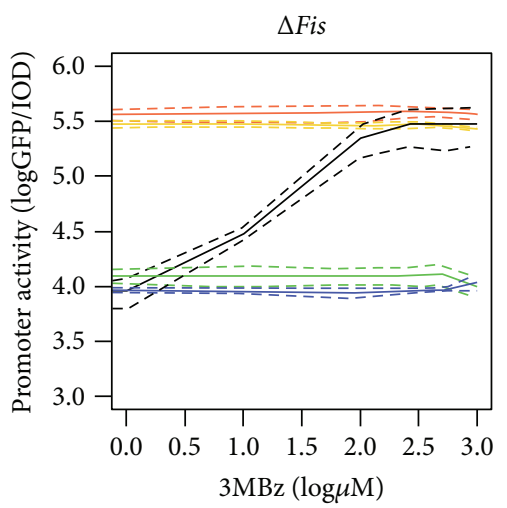

(d)

FIgure 4: The calibrator can be applied to the induction system xylS- $P m$. (a) xylS promoters (PxylS), xylS protein, and $P m$ promoter were cloned into the plasmid pMR1, which contains a short-lived GFP variant. (b) xylS-Pm calibration in LB solid medium with $1000 \mu \mathrm{M}$ of $3 \mathrm{MBz}$ added. This calibration was performed in BW25113wt strains. (c) Pjx promoter activity analyzed by 8 hours of experiment by monitoring GFPlva expression using pMR1 constructions. (d) GFP expression profile for 7 different $3 \mathrm{MBz}$ concentrations for Pjx and xylS- $P m$ in pMR1 vector, 4.5 hours after the induction. Solid lines represent the average values calculated using data from three independent experiments for wild type, $\Delta i h f$, and $\Delta f i s$ strains, while dashed lines represent the upper and lower limits of standard deviations.

to Pj106 promoter activity. Additionally, we can safely confirm that the sigmoidal form for the xylS-Pm system is due to the $3 \mathrm{MBz}$ addition and not by environmental or host changes, since the calibration system does not change under these conditions.

\section{Conclusions}

The standardization of biological parts for the construction of complex circuits forms the basis of synthetic biology [18-21]. In this regard, failure in the implementation of constructed synthetic biological circuits may occur when poorly characterized parts are used, and several strategies have been proposed to mitigate this problem [6, 22-24]. In this report, we have highlighted that simple experimental techniques involving the use of a single fluorescent reporter and plasmids are sufficient to provide robust characterization of transcriptional elements without the necessity of using of dual markers and complicated mathematical treatments [8]. Additionally, plasmids provide an easy way of implementing synthetic circuits that accelerates design-build-test cycles in synthetic biology. Once a circuit has been effectively implemented and tested, the introduction of a single copy of the construct by using a chromosome insertion on a same region for all promoters is recommended in order to enhance the performance of the system as well as provide stable strains for final use because an insertion on different regions could modify the GFP expression [25-27]. In this sense, the use of this promoter on different chromosome regions could provide a way to standardize the variation of gene expression caused by variations on chromosome position and structure. At the same time, the use of low-copy number plasmids can provide similar results as can the use of single-copy set-ups under certain circumstances $[1,15]$. In general, since each synthetic biology project has its own design and uses specific hosts and experimental conditions, the use of calibrators such as those described in this paper could provide a simple way to standardize the experimental conditions used. This would be similar to the use of molecular-weight size markers in molecular biology techniques as references for the 
comparison of samples of interest under varying experimental conditions. Although the calibration methods used in this study were implemented in strains of the Keio collection of E. coli mutants [12], we expect that the validations presented here will be adopted by other research groups studying synthetic biology as well as molecular microbiology. Additionally, the approaches used in this research study can be easily adopted using alternative plasmid standards for gram negative bacteria other than E. coli such as the vectors available at the SEVA database [16], thus creating a significant impact on research in microbiology.

\section{Conflicts of Interest}

The authors declare that there is no conflict of interest regarding the publication of this article.

\section{Acknowledgments}

This work was supported by the Young Investigator Award of Sao Paulo State Foundation (FAPESP, award number 2012/22921-8). Ananda Sanches-Medeiros was supported by a Scientific Initiation Scholarship (FAPESP, award number 2015/22386-3). Lummy Maria Oliveira Monteiro was supported by a FAPESP PhD fellowship (FAPESP, award number 2016/19179-9). The authors are thankful to the lab members for insightful discussions on this work.

\section{References}

[1] R. Silva-Rocha and V. de Lorenzo, "Chromosomal integration of transcriptional fusions," Methods in Molecular Biology, vol. 1149, pp. 479-489, 2014.

[2] A. Zaslaver, A. Bren, M. Ronen et al., "A comprehensive library of fluorescent transcriptional reporters for Escherichia coli," Nature Methods, vol. 3, no. 8, pp. 623-628, 2006.

[3] D. F. Browning and S. J. W. Busby, "Local and global regulation of transcription initiation in bacteria," Nature Reviews Microbiology, vol. 14, no. 10, pp. 638-650, 2016.

[4] L. Zelcbuch, N. Antonovsky, A. Bar-Even et al., "Spanning high-dimensional expression space using ribosome-binding site combinatorics," Nucleic Acids Research, vol. 41, no. 9, article e98, 2013.

[5] S. Klumpp and T. Hwa, "Bacterial growth: global effects on gene expression, growth feedback and proteome partition," Current Opinion in Biotechnology, vol. 28, pp. 96-102, 2014.

[6] J. R. Kelly, A. J. Rubin, J. H. Davis et al., "Measuring the activity of BioBrick promoters using an in vivo reference standard," Journal of Biological Engineering, vol. 3, no. 1, p. 4, 2009.

[7] B. Canton, A. Labno, and D. Endy, "Refinement and standardization of synthetic biological parts and devices," Nature Biotechnology, vol. 26, no. 7, pp. 787-793, 2008.

[8] T. J. Rudge, J. R. Brown, F. Federici et al., "Characterization of intrinsic properties of promoters," ACS Synthetic Biology, vol. 5, no. 1, pp. 89-98, 2016.

[9] M. Carbonell-Ballestero, E. Garcia-Ramallo, R. Montanez, C. Rodriguez-Caso, and J. Macia, "Dealing with the genetic load in bacterial synthetic biology circuits: convergences with the Ohm's law," Nucleic Acids Research, vol. 44, no. 1, pp. 496-507, 2016.
[10] J. B. Andersen, C. Sternberg, L. K. Poulsen, S. P. Bjorn, M. Givskov, and S. Molin, "New unstable variants of green fluorescent protein for studies of transient gene expression in bacteria," Applied and Environmental Microbiology, vol. 64, no. 6, pp. 2240-2246, 1998.

[11] A. Martinez-Antonio and J. Collado-Vides, "Identifying global regulators in transcriptional regulatory networks in bacteria," Current Opinion in Microbiology, vol. 6, no. 5, pp. 482489, 2003.

[12] T. Baba, T. Ara, M. Hasegawa et al., "Construction of Escherichia coli K-12 in-frame, single-gene knockout mutants: the Keio collection," Molecular Systems Biology, vol. 2, 2006.

[13] R. Silva-Rocha and V. de Lorenzo, "Broadening the signal specificity of prokaryotic promoters by modifying cis-regulatory elements associated with a single transcription factor," Molecular BioSystems, vol. 8, no. 7, pp. 1950-1957, 2012.

[14] M. E. Guazzaroni and R. Silva-Rocha, "Expanding the logic of bacterial promoters using engineered overlapping operators for global regulators," ACS Synthetic Biology, vol. 3, no. 9, pp. 666-675, 2014

[15] I. M. Benedetti, V. de Lorenzo, and R. Silva-Rocha, "Quantitative, non-disruptive monitoring of transcription in single cells with a broad-host range GFP-luxCDABE dual reporter system," PLoS One, vol. 7, no. 12, article e52000, 2012.

[16] R. Silva-Rocha, E. Martínez-García, B. Calles et al., "The Standard European Vector Architecture (SEVA): a coherent platform for the analysis and deployment of complex prokaryotic phenotypes," Nucleic Acids Research, vol. 41, no. D1, pp. D666-D675, 2013.

[17] A. Schmitz, "Cyclic AMP receptor protein interacts with lactose operator DNA," Nucleic Acids Research, vol. 9, no. 2, pp. 277-292, 1981.

[18] A. S. Khalil and J. J. Collins, "Synthetic biology: applications come of age," Nature Reviews Genetics, vol. 11, no. 5, pp. 367-379, 2010.

[19] R. P. Shetty, D. Endy, and T. F. Knight Jr, "Engineering BioBrick vectors from BioBrick parts," Journal of Biological Engineering, vol. 2, no. 1, p. 5, 2008.

[20] E. Andrianantoandro, S. Basu, D. K. Karig, and R. Weiss, "Synthetic biology: new engineering rules for an emerging discipline," Molecular Systems Biology, vol. 2, 2006.

[21] C. A. Voigt, "Genetic parts to program bacteria," Current Opinion in Biotechnology, vol. 17, no. 5, pp. 548-557, 2006.

[22] J. A. N. Brophy and C. A. Voigt, "Principles of genetic circuit design," Nature Methods, vol. 11, no. 5, pp. 508-520, 2014.

[23] V. Singh, "Recent advancements in synthetic biology: current status and challenges," Gene, vol. 535, no. 1, pp. 1-11, 2014.

[24] A. de Las Heras, C. A. Carreño, E. Martinez-Garcia, and V. de Lorenzo, "Engineering input/output nodes in prokaryotic regulatory circuits," FEMS Microbiology Reviews, vol. 34, no. 5, pp. 842-865, 2010.

[25] R. C. Brewster, F. M. Weinert, H. G. Garcia, D. Song, M. Rydenfelt, and R. Phillips, "The transcription factor titration effect dictates level of gene expression," Cell, vol. 156, no. 6, pp. 1312-1323, 2014.

[26] J. W. Lee, A. Gyorgy, D. E. Cameron et al., "Creating singlecopy genetic circuits," Molecular Cell, vol. 63, no. 2, pp. 329336, 2016. 
[27] D. F. Browning, D. C. Grainger, and S. J. W. Busby, "Effects of nucleoid-associated proteins on bacterial chromosome structure and gene expression," Current Opinion in Microbiology, vol. 13, no. 6, pp. 773-780, 2010.

[28] G. R. Amores, M. E. Guazzaroni, and R. Silva-Rocha, "Engineering synthetic cis-regulatory elements for simultaneous recognition of three transcriptional factors in bacteria," ACS Synthetic Biology, vol. 4, no. 12, pp. 1287-1294, 2015. 


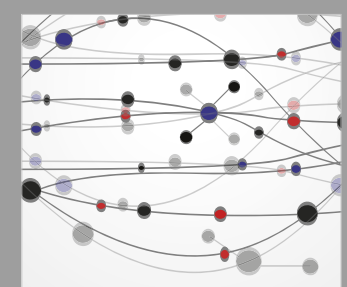

The Scientific World Journal
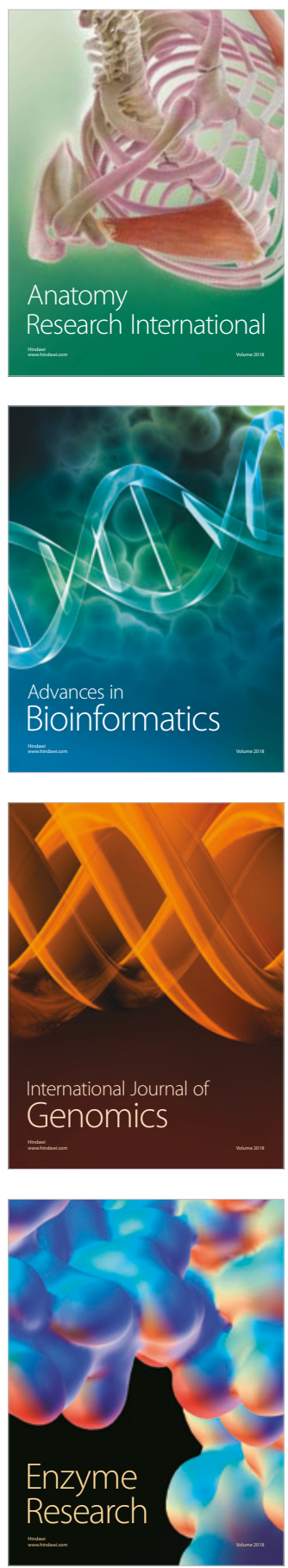
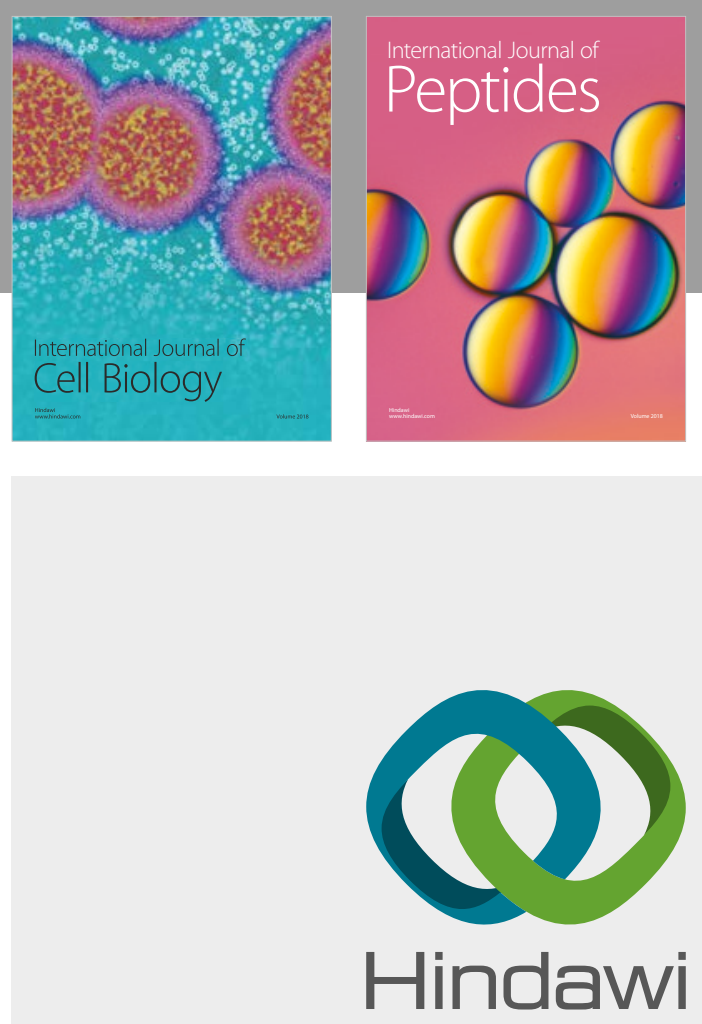

Submit your manuscripts at

www.hindawi.com
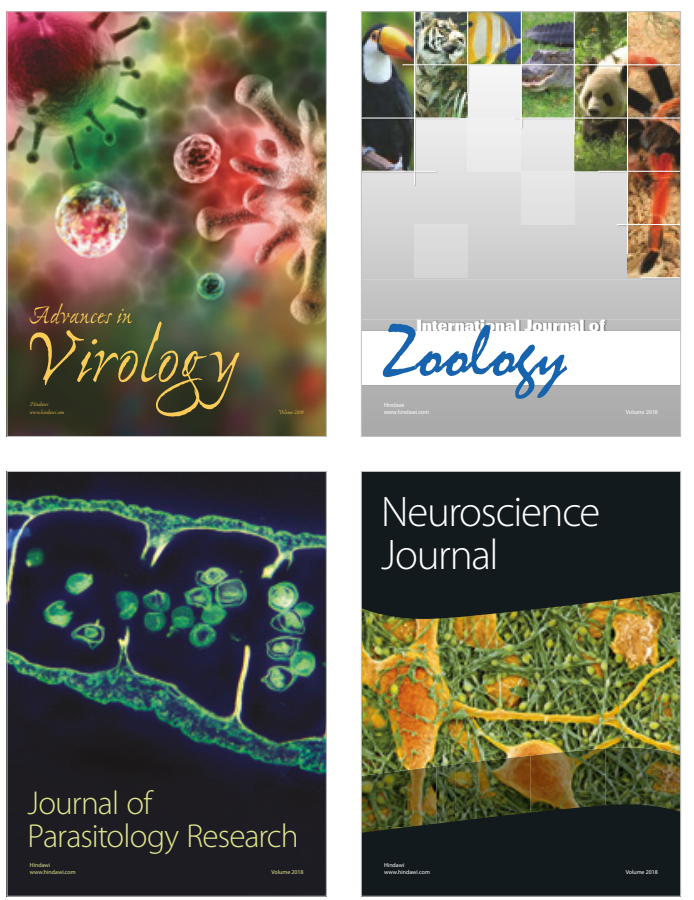
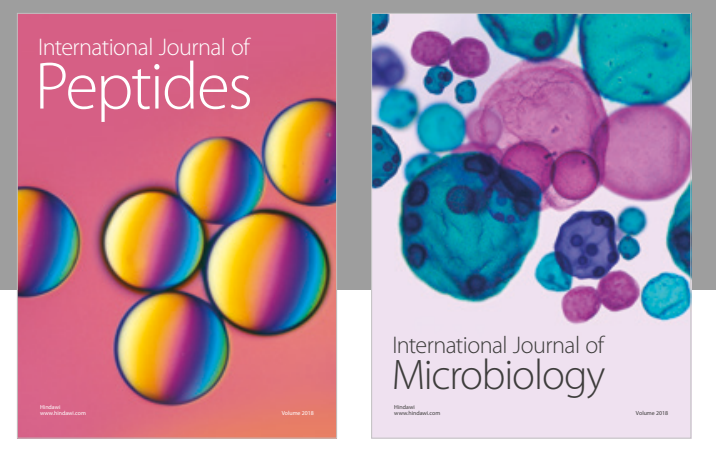

nternational Journal of Microbiology
Journal of
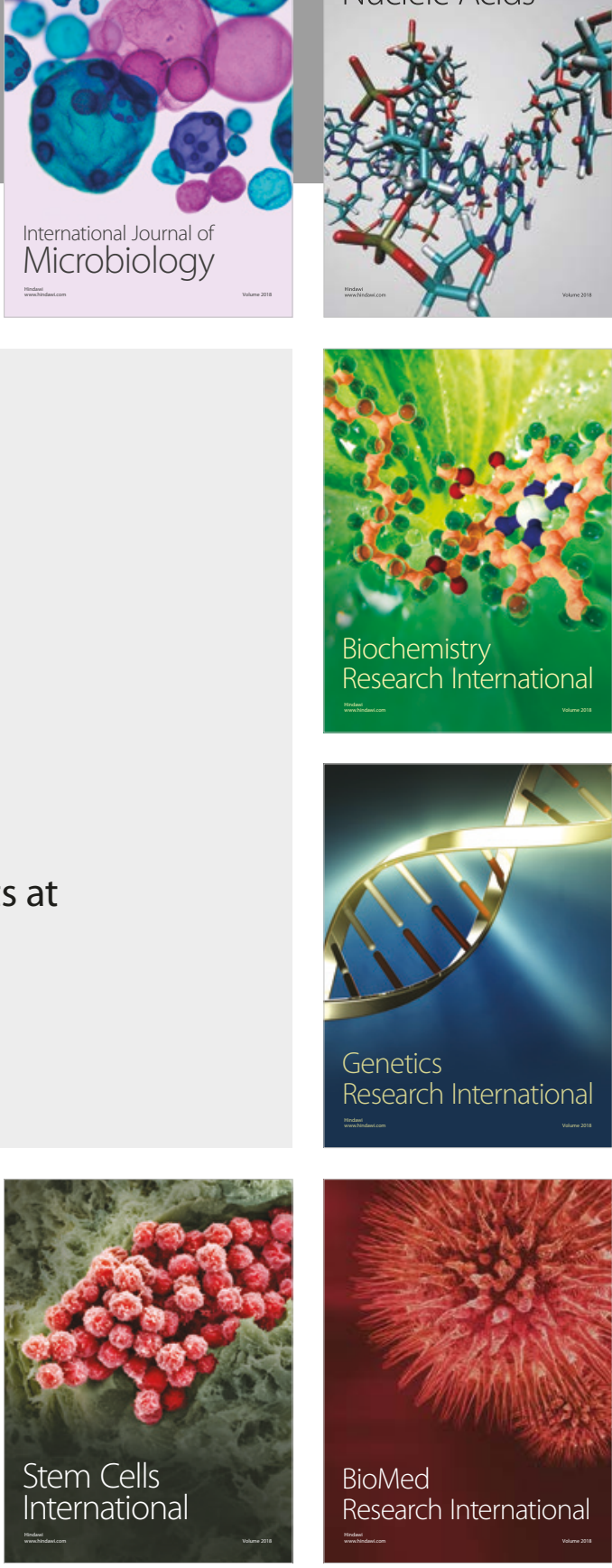
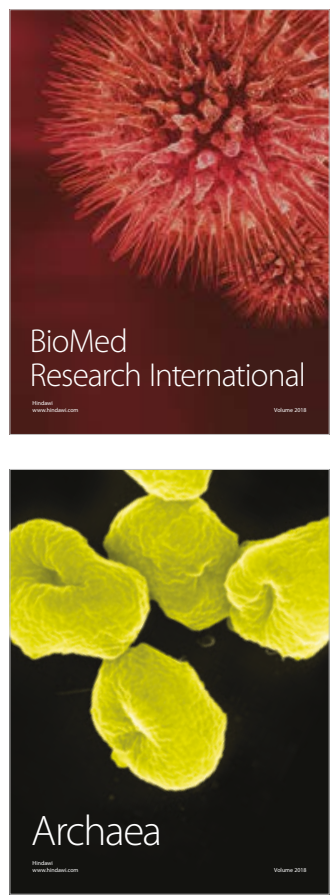